\title{
Mott transition in Kagomé lattice Hubbard model
}

\author{
Takuma Ohashi and Norio Kawakami \\ Department of Applied Physics, Osaka University, Suita, Osaka 565-0871, Japan \\ Hirokazu Tsunetsugu \\ Yukawa Institute for Theoretical Physics, Kyoto University, Kyoto 606-8502, Japan
}

(Dated: September 12, 2018)

\begin{abstract}
We investigate the Mott transition in the Kagomé lattice Hubbard model using a cluster extension of dynamical mean field theory. The calculation of the double occupancy, the density of states, the static and dynamical spin correlation functions demonstrates that the system undergoes the firstorder Mott transition at the Hubbard interaction $U / W \sim 1.4$ ( $W$ :bandwidth). In the metallic phase close to the Mott transition, we find the strong renormalization of three distinct bands, giving rise to the formation of heavy quasiparticles with strong frustration. It is elucidated that the quasiparticle states exhibit anomalous behavior in the temperature-dependent spin correlation functions.
\end{abstract}

PACS numbers: $71.30 .+\mathrm{h} 71.10 . \mathrm{Fd} 71.27 .+\mathrm{a}$

Geometrically frustrated electron systems have provided hot topics in the field of strongly correlated electron systems. The observation of heavy fermion behavior in $\mathrm{LiV}_{2} \mathrm{O}_{4}$ [1], which has the pyrochlore lattice structure with a corner-sharing network of tetrahedra, has activated theoretical studies of electron correlations with geometrical frustration. The discovery of superconductivity in the triangular lattice compound $\mathrm{Na}_{x} \mathrm{CoO}_{2} \cdot \mathrm{yH}_{2} \mathrm{O}$ [2] and the $\beta$-pyrochlore osmate $\mathrm{KOs}_{2} \mathrm{O}_{6}[3]$ has further stimulated intensive studies of frustrated electron systems. Geometrical frustration has uncovered new aspects of the Mott metal-insulator transition, which is now one of the central issues in the physics of strongly correlated electron systems. Among others, a novel quantum liquid ground state suggested for the Mott insulating phase of the triangular lattice [4] may be relevant for frustrated organic materials such as $\kappa-(\mathrm{ET})_{2} \mathrm{Cu}_{2}(\mathrm{CN})_{3}\left[\begin{array}{l}5 \\ \text { ] }\end{array}\right.$

The Kagomé lattice (Fig. 1) is another prototype of frustrated systems, which may be regarded as a twodimensional analog of the pyrochlore lattice. It is suggested that a correlated electron system on the Kagomé lattice can be an effective model of $\mathrm{Na}_{\mathrm{x}} \mathrm{CoO}_{2} \cdot \mathrm{yH}_{2} \mathrm{O}$ by properly considering anisotropic hopping matrix elements in the cobalt $3 d$ orbitals [6]. The issue of electron correlations for the Kagomé lattice was addressed recently by using the FLEX approximation [7] and QMC method [8]. These studies focused on electron correlations in the metallic regime, and the nature of the Mott transition has not been clarified yet. Therefore, it is desirable to investigate the Kagomé lattice electron system with particular emphasis on the Mott transition under the influence of strong frustration.

In this paper, we study the Mott transition of correlated electrons on the Kagomé lattice by means of the cellular dynamical mean field theory (CDFMT) 9]. It is shown that the metallic phase persists up to fairly large Coulomb interactions due to the frustrated lattice structure. This gives rise to the strong renormalization of

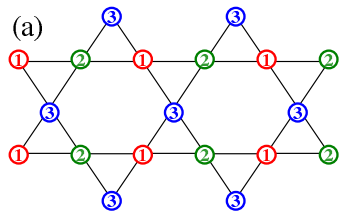

(b)

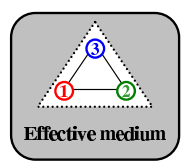

(c)

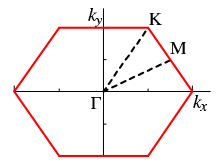

FIG. 1: (a) Sketch of the Kagomé lattice and (b) the effective cluster model using three sites cluster CDMFT. (c) First Brillouin zone of the Kagomé lattice.

three distinct bands, resulting in the multi-band quasiparticles with strong frustration near the Mott transition. In particular, we find that the quasiparticles exhibit anomalous behavior in spin correlation functions, which characterizes strong frustration in the metallic phase.

We consider the standard Hubbard model with nearest-neighbor hopping on the Kagomé lattice,

$$
H=-t \sum_{\langle i, j\rangle, \sigma} c_{i \sigma}^{\dagger} c_{j \sigma}+U \sum_{i} n_{i \uparrow} n_{i \downarrow}(t>0)
$$

with $n_{i \sigma}=c_{i \sigma}^{\dagger} c_{i \sigma}$, where $c_{i \sigma}^{\dagger}\left(c_{j \sigma}\right)$ creates (annihilates) an electron with spin $\sigma$ at site $i$. We use the band width $W=6 t$ as the energy unit. To study the Mott transition in the Kagomé lattice system, we need an efficient theoretical tool to treat both of strong correlations and geometrical frustration. The dynamical mean field theory (DMFT) 10 has given substantial theoretical progress in the field of the Mott transition but it does not incorporate spatially extended correlations. In order to treat both strong correlations and frustration, we use CDMFT, a cluster extension of DMFT, which has been successfully applied to frustrated systems such as the Hubbard model on the triangular lattice [11].

In CDMFT, the original lattice is regarded as a superlattice consisting of clusters, which is then mapped onto an effective cluster model via a standard DMFT procedure. Each unit cell of the Kagomé lattice has three sites 


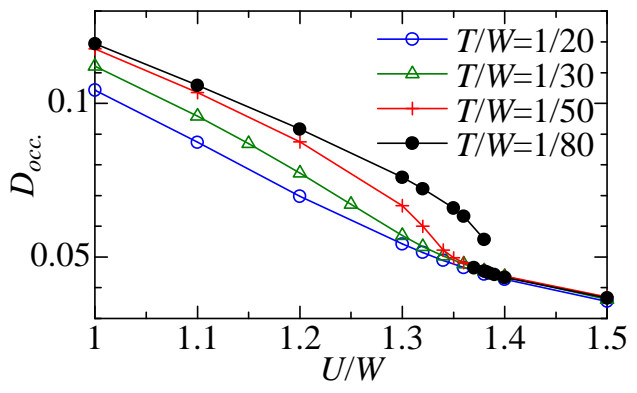

FIG. 2: Double occupancy as a function of interaction strength $U / W$ for several temperatures $T / W$. At $T / W=$ $1 / 80$, we can see the discontinuity with hysteresis, indicating the first-order Mott transition.

labeled by 1, 2, and 3, as shown in Fig. 1(a). We thus end up with a three-site cluster model coupled to the selfconsistently determined medium illustrated in Fig. 1(b). Given the Green's function for the effective medium, $\hat{\mathcal{G}}_{\sigma}$, we can compute the cluster Green's function $\hat{G}_{\sigma}$ and the cluster self-energy $\hat{\Sigma}_{\sigma}$ by solving the effective cluster model with QMC method [12]. Here, $\hat{\mathcal{G}}_{\sigma}, \hat{G}_{\sigma}$, and $\hat{\Sigma}_{\sigma}$ are described by $3 \times 3$ matrices. The effective medium $\hat{\mathcal{G}}_{\sigma}$ is then computed via the Dyson equation,

$\hat{\mathcal{G}}_{\sigma}^{-1}(\omega)=\left[\sum_{\mathbf{K}} \frac{1}{\omega+\mu-\hat{t}(\mathbf{K})-\hat{\Sigma}_{\sigma}(\omega)}\right]^{-1}+\hat{\Sigma}_{\sigma}(\omega)$,

where $\mu$ is the chemical potential. Here the summation of $\mathbf{K}$ is taken over the reduced Brillouin zone of the superlattice (see Fig. 1(c)) and $\hat{t}(\mathbf{K})$ is the Fouriertransformed hopping matrix for the superlattice. After twenty times iteration of this procedure, numerical convergence is reached. In each iteration, we typically use $10^{6}$ QMC sweeps and Trotter time slices $L=2 \mathrm{~W} / \mathrm{T}$ to reach sufficient computational accuracy. Furthermore, we exploit an interpolation scheme based on a high-frequency expansion of the discrete imaginary-time Green's function obtained by QMC [13] in order to reduce numerical errors resulting from the Fourier transformation from imaginary time to Matsubara frequency.

Let us now investigate the Mott transition of the Kagomé lattice Hubbard model at half filling. In Fig. 2 we show the results for the double occupancy $D_{\text {occ. }}=$ $\left\langle n_{i \uparrow} n_{i \downarrow}\right\rangle$ at various temperatures. At high temperatures, $D_{\text {occ. }}$ smoothly decreases as $U$ increases, indicating the development of local spin moments. As the temperature is lowered, there appears singular behavior around characteristic values of $U$. When $1 / 50 \leq T / W \leq 1 / 20, D_{\text {occ}}$. shows crossover behavior at $U / W \sim 1.35$. At lower temperature $T / W=1 / 80$, the crossover is changed to the discontinuity accompanied by hysteresis, which signals a first-order phase transition at $U_{c} / W \sim 1.37$. This is the first demonstration of the Mott transition in the Kagomé

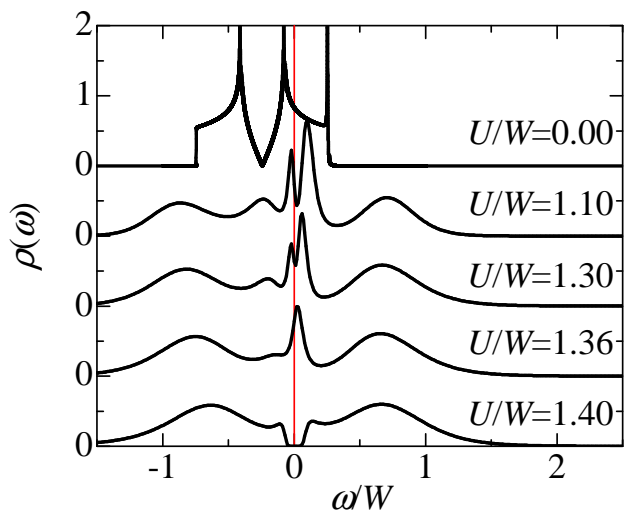

FIG. 3: Density of states at $T / W=1 / 80$ for several strengths of $U / W$.

lattice Hubbard model. Note that the critical interaction strength $U_{c}$ is much larger than the crossover strength of $U$ found for the unfrustrated square lattice 14. As is the case for the triangular-lattice Hubbard model [1]], the double occupancy $D_{\text {occ }}$. increases in the metallic phase $\left(U<U_{c}\right)$ as $T$ decreases, while it is almost independent of $T$ in the insulating phase $\left(U>U_{c}\right)$. The increase of $D_{\text {occ. }}$ at low temperatures means the suppression of the local moments due to the itinerancy of electrons, in other words, the formation of quasiparticles. Note that in the metallic phase close to the transition point, the increase of $D_{\text {occ. }}$ occurs at very low temperatures. This implies that the coherence temperature $T_{C}$ that characterizes the formation of quasiparticles is very low. This naturally causes strong frustration and, as shown below, brings about unusual metallic properties near the Mott transition.

To see how the quasiparticles evolve around the Mott transition point clearly, we calculate the density of states (DOS) by applying the maximum entropy method (MEM) 15] to the imaginary-time QMC data. In Fig. 3] we show DOS at $T / W=1 / 80$ for several values of the interaction $U / W$. In the non-interacting case $(U=0)$, DOS has three distinct bands including a $\delta$-function peak above the Fermi level. With increasing $U / W$, the DOS forms heavy quasiparticle peaks around the Fermi level and eventually develops a dip at $U / W \sim 1.40$, signaling the Mott transition. There are two characteristic properties in the metallic phase close to the critical point. First we note that heavy quasiparticles persist up to the transition point $(U / W=1.30$ and 1.36$)$ and there is no evidence for the pseudo-gap formation, which is consistent with the $U$ - and $T$-dependence of the double occupancy in Fig. 2. This is related to the suppression of magnetic instabilities in our system, in contrast to the square lattice case, where the quasiparticle states are strongly suppressed and a pseudo gap opens. Another point to be noticed is how strongly the renormalization occurs near the 


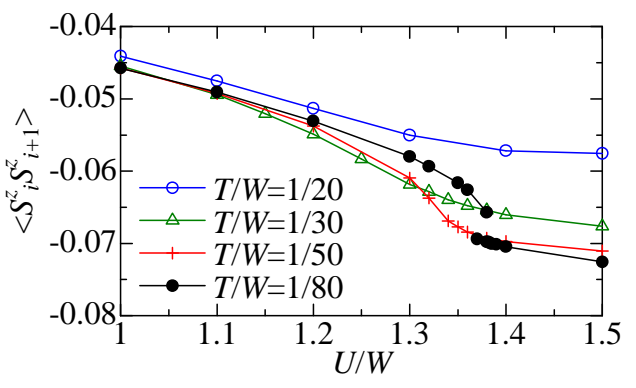

FIG. 4: The nearest neighbor spin correlation function $\left\langle S_{i}^{z} S_{i+1}^{z}\right\rangle$ as a function of $U / W$ at several temperatures. Note that the low-temperature spin correlation in the insulating phase is somewhat weaker than that for the isolated triangle, $\left\langle S_{i}^{z} S_{i+1}^{z}\right\rangle=-1 / 12$.

critical point. One can see three renormalized peaks near the Fermi level: not only the electrons near the Fermi surface but also the two bands away from the Fermi surface are renormalized to participate in the formation of quasiparticles. Therefore, the three quasiparticle bands are all relevant for low-energy excitations near the Mott transition, in contrast to the weak coupling regime where only the single band around the Fermi surface is relevant.

The remarkable fact we find is that the quasiparticles show anomalous properties in spin correlations due to strong frustration around the transition point. Shown in Fig. 4 is the nearest-neighbor spin correlation function $\left\langle S_{i}^{z} S_{i+1}^{z}\right\rangle$ at different temperatures. $\left\langle S_{i}^{z} S_{i+1}^{z}\right\rangle$ is always negative so that the spin correlation is antiferromagnetic (AF), which is a source of strong frustration. As $U / W$ increases, the nearest-neighbor AF spin correlation is enhanced gradually. In the insulating phase the AF spin correlation gets stronger with decreasing temperature, as is expected. We can see that more striking behavior emerges in the metallic phase close to the critical point: the AF spin correlation is once enhanced and then suppressed with the decrease of the temperature. The anomalous temperature dependence results from the competition between the quasiparticle formation and the frustrated spin correlations, which may be characterized by two energy scales: the coherence temperature $T_{C}$ and $T_{M}$ characterizing the AF spin fluctuations. The AF correlation is developed around $T \sim T_{M}$, which stabilizes localized moments and causes frustration in accordance with the monotonic enhancement of spin correlations in the insulating phase in Fig. 4 On the other hand, when the system is in the metallic phase, electrons recover coherence in itinerant motion below $T_{C}$. Therefore, the frustration is relaxed by itinerancy of electrons via the suppression of AF correlations at $T<T_{C}$. Thus, the nonmonotonic temperature-dependence of $\left\langle S_{i}^{z} S_{i+1}^{z}\right\rangle$ clearly demonstrates that the heavy quasiparticles are formed under the influence of strong frustration.

The anomalous properties also appear in dynamical

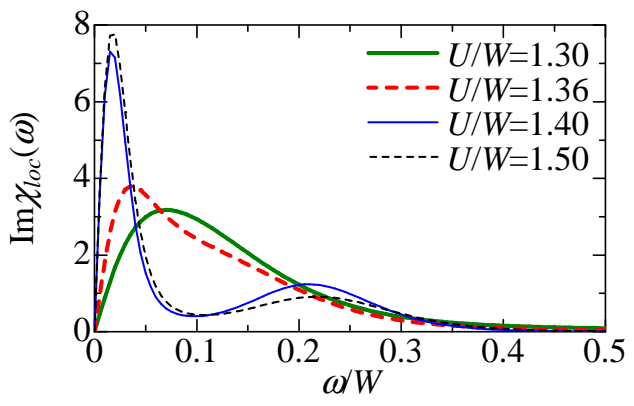

FIG. 5: Dynamical susceptibility $\operatorname{Im} \chi_{l o c}(\omega)$ at $T / W=1 / 80$ for several strengths of $U / W$.

spin correlation functions. We calculate the dynamical spin susceptibility $\chi_{l o c}(\omega)=-i \int d t e^{i \omega t}\left\langle\left[S_{i}^{z}(t), S_{i}^{z}(0)\right]\right\rangle$, where $S_{i}^{z}=\left(c_{i \uparrow}^{\dagger} c_{i \uparrow}-c_{i \downarrow}^{\dagger} c_{i \downarrow}\right) / 2$. In Fig. [5] we show $\operatorname{Im} \chi_{l o c}(\omega)$ around the Mott transition at $T / W=1 / 80$. A remarkable point is that the profile of $\operatorname{Im} \chi_{l o c}(\omega)$ dramatically changes around the Mott transition. In the insulating phase $(U / W=1.4,1.5), \operatorname{Im} \chi_{l o c}(\omega)$ has two distinct peaks at low energies. In the metallic phase $(U / W=1.3,1.36)$, two peaks get renormalized into a single peak and its peak value is strongly suppressed. This is the first demonstration of drastic change of spin dynamics between metallic and insulating phases in frustrated systems. In the insulating phase, the short-range AF correlations become dominant at low temperatures, resulting in the appearance of the double-peak structure. The strongly enhanced low-energy peak in $\chi_{\text {loc }}(\omega)$ corresponds to excitations among the almost degenerate states for which a singlet spin pair is formed inside the unit cell, while the higher-energy hump is caused by the excitations from these low-energy states to other excited states. In the metallic phase, the AF correlations are suppressed and then frustration is relaxed via the itinerancy of electrons, which leads to the renormalized single peak structure in $\chi_{l o c}(\omega)$. Therefore, the dramatic change in $\chi_{l o c}(\omega)$ features the competition between itinerancy and frustration of correlated electrons around the Mott transition point.

Finally, we discuss the magnetic instability by examining the wavevector-dependence of the static susceptibility,

$$
\begin{aligned}
& \chi_{\gamma \delta}(\mathbf{q})=\int_{0}^{1 / T} d \tau \sum_{\mathbf{k}, \mathbf{k}^{\prime}} \\
& \times\left\langle c_{\mathbf{k} \gamma \uparrow}^{\dagger}(\tau) c_{\mathbf{k}+\mathbf{q} \gamma \downarrow}(\tau) c_{\mathbf{k}^{\prime}+\mathbf{q} \delta \downarrow}^{\dagger}(0) c_{\mathbf{k}^{\prime} \delta \uparrow}(0)\right\rangle
\end{aligned}
$$

where $\gamma, \delta=1,2,3$ denote the superlattice indices. We employ the standard procedure in DMFT to calculate $\chi_{\gamma \delta}(\mathbf{q})$ [10], which includes nearest-neighbor correlations as well as on-site correlations. It is convenient to introduce $\chi_{m}(\mathbf{q})$ for three normal modes $(m=1,2,3)$ by diagonalizing the $3 \times 3$ matrix $\chi_{\gamma \delta}(\mathbf{q})$. We find that the 


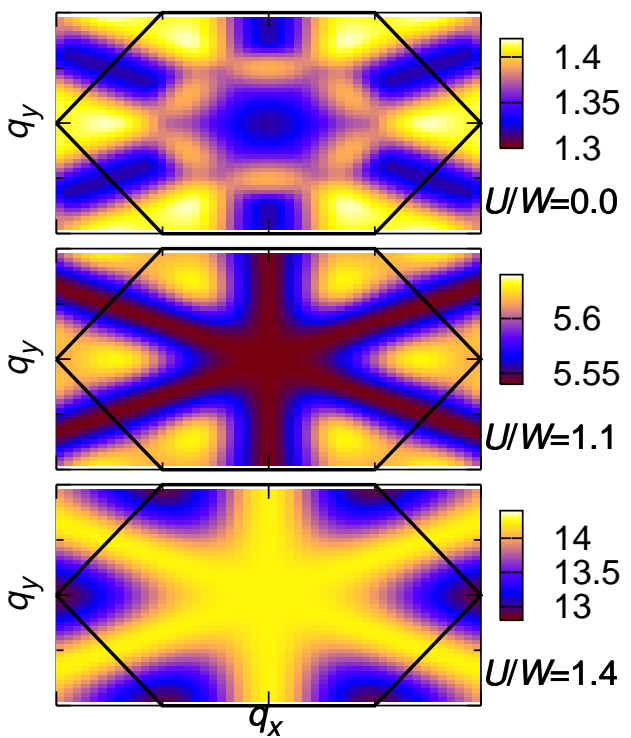

FIG. 6: The maximum mode of the susceptibility $\chi_{\max }(\mathbf{q})$ for different strength of $U / W$ at $T / W=1 / 30$. Hexagons in figures denotes the first Brillouin zone as shown Fig. 1] (c).

q-dependence of the mode with the maximum eigenvalue (referred to as $\chi_{\max }(\mathbf{q})$ ) is much weaker than that for the other two modes, while the second largest mode has the strong $\mathbf{q}$-dependence with a maximum at $\mathbf{q}=(0,0)$. These results are consistent with the previous FLEX calculation [7] and the QMC study [8]. We find, however, notable new results in the strong coupling regime. In Fig. [6] we show $\chi_{\max }(\mathbf{q})$ for several strengths of $U / W$ at $T / W=1 / 30$. In the noninteracting case $U / W=0$, the susceptibility takes a maximum at six points in the Brillouin zone. As $U / W$ increases, the susceptibility is enhanced not only at these six points but also on the lines through $\Gamma$ and $\mathrm{M}$ points, so that $\chi_{\max }(\mathbf{q})$ becomes much flatter at $U / W=1.1$ than in the noninteracting case. Once the system enters the insulating phase, the q-dependence of $\chi_{\max }(\mathbf{q})$ dramatically changes its character due to the enhancement of short range AF correlations. At $U / W=1.4$, the susceptibility is further enhanced along the three lines in $\mathbf{q}$ space and becomes dominant instead of the six points that give the leading magnetic mode in the weak coupling regime. Furthermore, by investigating the eigenvectors of $\chi_{\max }(\mathbf{q})$, we find that two spins in the unit cell are antiferromagnetically coupled but the other spin is free. Therefore, these enhanced spin fluctuations favor a spatial spin configuration in which one-dimensional AF-correlated spin chains are independently formed in three distinct directions. This is consistent with the intra chain spin correlations in the $\mathbf{q}=0$ structure predicted for the Kagomé Heisenberg systems [16]. However, the essential difference is that there is almost no correlation between different chains. This is the first observation of one-dimensional spin correlations in the itinerant electron systems with geometrical frustration. Although we have not obtained real instability to a novel one-dimensional ordering in the present calculation, such enhanced spin fluctuations certainly affect low-energy dynamics in the insulating phase.

In summary we have investigated the Mott transition in the Kagomé lattice Hubbard model using CDMFT combined with QMC. We have found that the metallic phase is stabilized up to fairly large $U$, resulting in the three-band heavy quasiparticles with strong frustration. This causes several anomalous properties of spin correlation functions in the metallic phase close to the critical point. We have also discussed the possibility of magnetic instability toward a novel one-dimensional ordering in the insulating phase.

The authors thank S. Suga, Y. Motome, A. Koga, and Y. Imai for valuable discussions. A part of numerical computations was done at the Supercomputer Center at the Institute for Solid State Physics, University of Tokyo. This work was partly supported by a Grant-in-Aid from the Ministry of Education, Science, Sports and Culture of Japan.

[1] S. Kondo, et al., Phys. Rev. Lett. 78, 3729 (1997).

[2] K. Takada, et al., Nature (London) 422, 53 (2003).

[3] S. Yonezawa, Y. Muraoka, Y. Matsushita, and Z. Hiroi, J. Phys.: Cond. Mat. 16, L9 (2004).

[4] T. Kashima, and M. Imada, J. Phys. Soc. Jpn. 70, 3052 (2001).

[5] Y. Shimizu, K. Miyagawa, K. Kanoda, M. Maesato, and G. Saito, Phys. Rev. Lett. 91, 107001 (2003).

[6] W. Koshibae and S. Maekawa, Phys. Rev. Lett. 91, 257003 (2003).

[7] Y. Imai, N. Kawakami, and H. Tsunetsugu Phys. Rev. B 68, 195103 (2003).

[8] N. Bulut, W. Koshibae, and S. Maekawa Phys. Rev. Lett. 95, 037001 (2005).

[9] G. Kotliar, S. Y. Savrasov, G. Pálsson, and G. Biroli Phys. Rev. Lett. 87, 186401 (2001).

[10] A. Georges, G. Kotliar, W. Krauth and M. J. Rozenberg, Rev. Mod. Phys. 68, 13 (1996).

[11] O. Parcollet, G. Biroli, and G. Kotliar, Phys. Rev. Lett. 92, 226402, (2004).

[12] J. E. Hirsch and R. M. Fye, Phys. Rev. Lett. 56, 2521 (1986).

[13] V. S. Oudovenko and G. Kotliar Phys. Rev. B 65, 075102 (2002).

[14] S. Moukouri, and M. Jarrell Phys. Rev. Lett. 87, 167010, (2001).

[15] M. Jarrell and J. E. Gubernatis, Phys. Rep. 269, 133 (1996).

[16] A. B. Harris, C. Kallin, and A. J. Berlinsky, Phys. Rev. B 45, 2899 (1992). 